\title{
On support of solutions to singular nonlinear parabolic equations in bounded domains
}

\author{
FABIO PUNZO \\ Dipartimento di Matematica "G. Castelnuovo”, Università di Roma "La Sapienza”, \\ P.le A. Moro 5, I-00185 Roma, Italy \\ E-mail: punzo@mat.uniroma1.it
}

[Received 25 June 2010 and in revised form 6 August 2010]

\begin{abstract}
We investigate support properties of nonnegative solutions to nonlinear parabolic equations with variable density in bounded domains. The density can diverge or vanish near the boundary. Assuming that the initial datum has support not intersecting the boundary, we provide simple conditions, in dependence on the behaviour of the density, guaranteeing that the support of every nonnegative solution intersects the boundary at some positive time, or, in the case of convex domains, that it remains away from it for any positive time. These results extend to the case of bounded domains those given in $[\mathrm{KK}]$ for the Cauchy problem.
\end{abstract}

2010 Mathematics Subject Classification: Primary 35K61, 35K67, 35B99; Secondary 35B40, 35B51.

Keywords: Support of solutions; sub- and supersolutions; comparison principles.

\section{Introduction}

We consider nonnegative bounded solutions to nonlinear parabolic equations of the following type:

$$
\rho \partial_{t} u=\Delta[G(u)] \quad \text { in } \Omega \times(0,+\infty)=: Q,
$$

where $\Omega$ is an open bounded subset of $\mathbb{R}^{n}(n \geqslant 1)$ with boundary $\partial \Omega \equiv \mathcal{S}$, and $\rho=\rho(x)$, which will be referred to as a density, is a positive function only depending on the space variable; moreover, a typical choice for the function $G$ is $G(u)=u^{m}, m>1$.

Following [KT], [PT], [P2], we allow the density $\rho$ to vanish, to diverge, or to not have a limit as the distance $\operatorname{dist}(x, \mathcal{S}) \equiv d(x)$ goes to zero. On the other hand, $\rho$ is supposed to be positive and continuous inside $\Omega$. More precisely, we always make the following assumptions:

$\left(H_{1}\right) \quad \mathcal{S}$ is an $(n-1)$-dimensional compact submanifold of $\mathbb{R}^{n}$ of class $C^{3}$,

(i) $\rho \in C(\Omega), \rho>0$ in $\Omega$;

(ii) $G \in C^{1}\left([0,+\infty), G(0)=G^{\prime}(0)=0, G^{\prime}(s)>0\right.$ for any $s>0$, $G^{\prime}$ is increasing in $[0, \delta]$ for some $\delta>0$;

(iii) $u_{0} \in C(\Omega), u_{0} \geqslant 0, \operatorname{supp} u_{0} \cap \mathcal{S}=\emptyset$.

Hence, it is natural to study the following initial value problem:

$$
\begin{cases}\rho \partial_{t} u=\Delta[G(u)] & \text { in } Q, \\ u=u_{0} & \text { in } \Omega \times\{0\}\end{cases}
$$

notice that in (1.1) no boundary conditions are imposed at $\mathcal{S}$. 
The well-posedness of problem (1.1) has been studied in [KT] for $n=1$ and in [P2] for $n \geqslant 1$. If $\rho(x) \rightarrow 0$ fast enough as $d(x) \rightarrow 0$ then nonuniqueness of bounded solutions is proved; on the contrary, when $\rho$ does not vanish at $\mathcal{S}$, or $\rho(x) \rightarrow 0$ slowly as $d(x) \rightarrow 0$, then uniqueness of bounded solutions not satisfying any extra conditions at $\mathcal{S}$ is showed.

The aim of this paper is to investigate support properties of nonnegative bounded solutions to problem (1.1), in dependence on the behaviour of $\rho$ near the boundary $\mathcal{S}$; special attention will be paid to the case $G(u)=u^{m}(m>1)$, when problem (1.1) reads

$$
\begin{cases}\rho \partial_{t} u=\Delta\left(u^{m}\right) & \text { in } Q, \\ u=u_{0} & \text { in } \Omega \times\{0\} .\end{cases}
$$

In particular, we shall prove that if $\rho \in L^{1}(\Omega)$ and

$$
\int_{0}^{1} \frac{G^{\prime}(s)}{s} \mathrm{~d} s<+\infty
$$

then for every nonnegative solution $u$ to problem $(1.1), \operatorname{supp} u\left(\cdot, t_{0}\right)$ intersects $\mathcal{S}$ for some $t_{0}>0$. Moreover, when $G(u)=u^{m}(m>1)$, the hypothesis $\rho \in L^{1}(\Omega)$ can be replaced by the weaker condition $\rho[d(x)]^{\alpha / m} \in L^{1}(\Omega)$ for some $\alpha \in(0,1)$. Instead, if $\Omega$ is convex and $\rho(x) \geqslant C[d(x)]^{-\alpha} \quad(x \in \Omega)$ for some $C \geqslant 0$ and $\alpha \geqslant 2$, then for every nonnegative solution $u$ to problem (1.1), supp $u(\cdot, t)$ does not intersect $\mathcal{S}$ for any $t>0$.

Similar results have already been proved in [KK] (see also [GHP]) for the Cauchy problem

$$
\begin{cases}\rho \partial_{t} u=\Delta[G(u)] & \text { in } \mathbb{R}^{n} \times(0,+\infty), \\ u=u_{0} & \text { in } \mathbb{R}^{n} \times\{0\},\end{cases}
$$

supposing that the functions $\rho, u_{0}, G$ satisfy the hypothesis

(i) $\rho \in C^{1}\left(\mathbb{R}^{n}\right) \cap L^{\infty}\left(\mathbb{R}^{n}\right), \rho>0$;

(ii) $G \in C^{1}([0,+\infty)), G(0)=G^{\prime}(0)=0, G^{\prime}(s)>0$ for any $s>0$, $G^{\prime}$ is increasing in $[0, \delta]$ for some $\delta>0$;

(iii) $u_{0} \in C\left(\mathbb{R}^{n}\right), u_{0} \geqslant 0$, supp $u_{0}$ is compact.

To be specific, in $[\mathrm{KK}]$ it has been proved that if $n \geqslant 3, \rho \in L^{1}\left(\mathbb{R}^{n}\right)$ and $\left(H_{3}\right)$ holds true, then for every nonnegative bounded solution $u$ to problem (1.3) there exists $t_{0}>0$ such that supp $u\left(\cdot, t_{0}\right)$ is not compact. Moreover, when $G(u)=u^{m}(m>1)$, the assumption $\rho \in L^{1}\left(\mathbb{R}^{n}\right)$ can be replaced by the weaker condition $\rho|x|^{(2-n) / m} \in L^{1}\left(\mathbb{R}^{n}\right)$. On the contrary, when $n \geqslant 1, G(u)=u^{m}(m>1)$ and

$$
\rho(x) \geqslant \frac{C}{\left(1+|x|^{2}\right)^{\alpha / 2}} \quad\left(x \in \mathbb{R}^{n}\right),
$$

for some $0<\alpha \leqslant 2$ and $C>0$, then for every nonnegative bounded solution $u$ to problem (1.3) $\operatorname{supp} u(\cdot, t)$ remains compact for any $t>0$.

In connection with these results of [KK] let us mention that if $n \geqslant 3$ and $\rho \rightarrow 0$ fast enough as $|x| \rightarrow \infty$, then nonuniqueness of bounded solutions to problem (1.3) has been proved (see [EK], [KKT], [P1]). Instead, when $n=2$ or $n \geqslant 3$ and condition (1.4) holds true, then uniqueness for problem (1.3), in the class of bounded solutions not satisfying any extra constraints at infinity, has been showed (see [KKT], [P1]). 
Roughly speaking, for problem (1.1) the boundary $\mathcal{S}$ plays the same role as infinity for problem (1.3). Hence, to study problem (1.1), the requirement supp $u_{0} \cap \mathcal{S}=\emptyset$ in assumption $\left(H_{2}\right)$ (iii) corresponds to the requirement that $\operatorname{supp} u_{0}$ is compact in $\left(H_{4}\right)$ (iii) to study problem (1.3).

Clearly, results that we shall prove can be regarded as an extension to problem (1.1) of results given in [KK] for problem (1.3).

\subsection{Results}

More precisely, we shall prove the following results.

THEOREM 1.1 Let assumptions $\left(H_{1}\right)-\left(H_{3}\right)$ be satisfied, and $\rho \in L^{1}(\Omega)$. Let $u$ be any solution to problem (1.1). Then there exists $t_{0}>0$ such that $\operatorname{supp} u\left(\cdot, t_{0}\right) \cap \mathcal{S} \neq \emptyset$.

COROLlary 1.2 Let assumptions $\left(H_{1}\right)$ and $\left(H_{2}\right)(\mathrm{i})$,(iii) be satisfied; suppose that $G(u)=u^{m}$ $(m>1)$ and $\rho[d(x)]^{\alpha / m} \in L^{1}(\Omega)$ for some $\alpha \in(0,1)$. Let $u$ be any solution to problem (1.1). Then there exists $t_{0}>0$ such that $\operatorname{supp} u\left(\cdot, t_{0}\right) \cap \mathcal{S} \neq \emptyset$.

Let $\mathcal{S}^{\varepsilon}:=\{x \in \Omega \mid \mathrm{d}(x)<\varepsilon\}(\varepsilon>0)$.

THEOREM 1.3 Let assumptions $\left(H_{1}\right)$ and $\left(H_{2}\right)$ (i), (iii) be satisfied; suppose that $\Omega$ is convex and that $G(u)=u^{m}(m>1)$. Assume that there exist $C>0, \tilde{\varepsilon}>0$ and $\alpha \geqslant 2$ such that

$$
\rho(x) \geqslant \frac{C}{[d(x)]^{\alpha}} \quad\left(x \in \mathcal{S}^{\tilde{\varepsilon}}\right) .
$$

Let $u$ be any solution to problem (1.1). Then $\operatorname{supp} u(\cdot, t) \cap \mathcal{S}=\emptyset$ for any $t>0$. Moreover,

(i) if $\alpha>2$, then there exist $a>0, b>0, \hat{\varepsilon}>0$ such that, for any $t>0$,

$$
\operatorname{supp} u(\cdot, t) \subseteq\left(\Omega \backslash \mathcal{S}^{\hat{\varepsilon}}\right) \cup\left\{x \in \mathcal{S}^{\hat{\varepsilon}} \mid d(x, \mathcal{S})>\frac{1}{a(b t+1)^{1 /(\alpha-2)}}\right\} ;
$$

(ii) if $\alpha=2$, then there exist $a>0, \beta>0, \hat{\varepsilon}>0$ such that, for any $t>0$,

$$
\operatorname{supp} u(\cdot, t) \subseteq\left(\Omega \backslash \mathcal{S}^{\hat{\varepsilon}}\right) \cup\left\{x \in \mathcal{S}^{\hat{\varepsilon}} \mid d(x, \mathcal{S})>\frac{1}{a e^{\beta t}}\right\} .
$$

In a forthcoming paper we shall study if Theorem 1.3 can be generalized to the case of a nonconvex domain $\Omega$.

\section{Mathematical framework}

DEFINITION 2.1 By a solution of problem (1.1) we mean a nonnegative function $u \in C(Q) \cap$ $L^{\infty}(Q)$ such that

$$
\begin{aligned}
\int_{0}^{\tau} \int_{\Omega_{1}}\left\{\rho u \partial_{t} \psi+G(u) \Delta \psi\right\} \mathrm{d} x \mathrm{~d} t= & \int_{\Omega_{1}} \rho\left[u(x, \tau) \psi(x, \tau)-u_{0}(x) \psi(x, 0)\right] \mathrm{d} x \\
& +\int_{0}^{\tau} \int_{\partial \Omega_{1}} G(u)\langle\nabla \psi, v\rangle \mathrm{d} \sigma \mathrm{d} t
\end{aligned}
$$


for any open set $\Omega_{1} \subseteq \Omega$ with smooth boundary, $\overline{\Omega_{1}} \subseteq \Omega, \tau>0, \psi \in C^{2,1}\left(\overline{\Omega_{1}} \times[0, \tau]\right), \psi \geqslant 0$, $\psi=0$ in $\partial \Omega_{1} \times[0, \tau]$; here $v$ denotes the outer normal to $\Omega_{1}$ and $\langle\cdot, \cdot\rangle$ the scalar product in $\mathbb{R}^{n}$.

Supersolutions (or subsolutions) of (1.1) are defined by replacing "=" by " $\leqslant$ " (" $\geqslant "$, respectively) in (2.1).

We will also consider, for any $\varepsilon>0$ small enough, the auxiliary problem

$$
\begin{cases}\rho u_{t}=\Delta[G(u)] & \text { in }\left[\Omega \backslash \overline{\mathcal{S}^{\varepsilon}}\right] \times(0,+\infty)=: Q_{\varepsilon}, \\ u=0 & \text { in } \mathcal{A}^{\varepsilon} \times(0,+\infty), \\ u=u_{0} & \text { in }\left[\Omega \backslash \overline{\mathcal{S}^{\varepsilon}}\right] \times\{0\}\end{cases}
$$

here $\mathcal{A}^{\varepsilon}:=\{x \in \Omega \mid \mathrm{d}(x)=\varepsilon\}$.

DEFinition 2.2 By a supersolution of problem (2.2) we mean a nonnegative function $u \in$ $C\left(Q_{\varepsilon}\right) \cap L^{\infty}\left(Q_{\varepsilon}\right)$ such that

$$
\begin{aligned}
\int_{0}^{\tau} \int_{\Omega_{1}}\left\{\rho u \partial_{t} \psi+G(u) \Delta \psi\right\} \mathrm{d} x \mathrm{~d} t \leqslant & \int_{\Omega_{1}} \rho\left[u(x, \tau) \psi(x, \tau)-u_{0}(x) \psi(x, 0)\right] \mathrm{d} x \\
& +\int_{0}^{\tau} \int_{\partial \Omega_{1} \backslash \mathcal{A}^{\varepsilon}} G(u)\langle\nabla \psi, v\rangle \mathrm{d} \sigma \mathrm{d} t
\end{aligned}
$$

for any open set $\Omega_{1} \subseteq \Omega \backslash \overline{\mathcal{S}^{\varepsilon}}$ with smooth boundary, $\tau \in(0, T], \psi \in C^{2,1}\left(\overline{\Omega_{1}} \times[0, \tau]\right), \psi \geqslant 0$, $\psi=0$ in $\partial \Omega_{1} \times[0, \tau]$. Solutions and subsolutions are defined accordingly.

For further purposes, let us also introduce the elliptic equation

$$
\Delta U=f \quad \text { in } \Omega,
$$

where $f \in C(\Omega)$, and the elliptic problem

$$
\begin{cases}\Delta U=f & \text { in } \Omega \backslash \overline{\mathcal{S}^{\varepsilon}} \\ U=0 & \text { in } \mathcal{A}^{\varepsilon}\end{cases}
$$

where $f \in C\left(\Omega \backslash \overline{\mathcal{S}^{\varepsilon}}\right)$.

Definition 2.3 By a supersolution to equation (2.3) we mean a function $U \in C(\Omega)$ such that

$$
\int_{\Omega_{1}} U \Delta \psi \mathrm{d} x \leqslant \int_{\partial \Omega_{1}} U\langle\nabla \psi, v\rangle \mathrm{d} \sigma+\int_{\Omega_{1}} f \psi \mathrm{d} x
$$

for any open set $\Omega_{1} \subseteq \Omega$ with smooth boundary, $\overline{\Omega_{1}} \subseteq \Omega, \psi \in C^{2}\left(\overline{\Omega_{1}}\right), \psi \geqslant 0, \psi=0$ in $\partial \Omega_{1}$; here $v$ denotes the outer normal to $\Omega_{1}$. Subsolutions and solutions of problem (2.3) are defined accordingly.

Definition 2.4 By a supersolution of problem (2.4) we mean a function $U \in C\left(\bar{\Omega} \backslash \mathcal{S}^{\varepsilon}\right)$ such that

$$
\int_{\Omega_{1}} U \Delta \psi \mathrm{d} x \leqslant \int_{\partial \Omega_{1} \backslash \mathcal{A}^{\varepsilon}} U\langle\nabla \psi, v\rangle \mathrm{d} \sigma+\int_{\Omega_{1}} f \psi \mathrm{d} x
$$

for any open set $\Omega_{1} \subseteq \Omega \backslash \overline{\mathcal{S}^{\varepsilon}}$ with smooth boundary, $\psi \in C^{2}\left(\overline{\Omega_{1}}\right), \psi \geqslant 0, \psi=0$ in $\partial \Omega_{1}$; here $v$ denotes the outer normal to $\Omega_{1}$. Solutions and subsolutions are defined accordingly. 
We need some preliminary material concerning the distance function $x \mapsto d(x)(x \in \Omega)$. First, observe that in view of the compactness and regularity of $\mathcal{S}$ assumed in $\left(H_{1}\right)$, there exists $\sigma>0$ such that for any $x \in \mathcal{S}^{\sigma}$ there exists a unique point $x^{*}(x) \in \mathcal{S}$ such that $d(x)=\left|x-x^{*}(x)\right|$; moreover (see $[\mathrm{F}]), x^{*}(\cdot) \in C^{2}\left(\mathcal{S}^{\sigma} ; \mathcal{S}\right), \mathrm{d} \in C^{3}\left(\overline{\mathcal{S}^{\sigma}}\right)$ and (see also $[\mathrm{A}]$ )

$$
|\nabla d(x)|=1 \quad \text { for any } x \in \mathcal{S}^{\sigma} .
$$

Furthermore, when $\Omega \subseteq \mathbb{R}^{n}$ is a convex subset, then (see e.g. [AD])

$$
\Delta d(x) \leqslant 0 \quad \text { for any } x \in \mathcal{S}^{\sigma} .
$$

For any $x_{0} \in \mathbb{R}^{n}, R>0$ we set $B_{R}\left(x_{0}\right):=\left\{x \in \mathbb{R}^{n}|| x-x_{0} \mid<R\right\}$.

Let $y^{0} \in \mathcal{S}$; let $T_{y^{0}} \mathcal{S}$ and $\perp_{y^{0}} \mathcal{S}$ denote respectively the tangent and the orthogonal space to $\mathcal{S}$ at $y^{0}$. For further purposes, observe that we can choose a new coordinate system $X \equiv\left(X_{1}, \ldots, X_{n}\right)$ in $\mathbb{R}^{n}$ such that, if $p \equiv p_{n}: B_{R}(0) \subseteq \mathbb{R}^{n-1} \rightarrow \mathbb{R}(R>0)$ denotes the local representation of $\mathcal{S}$ near $X^{*}\left(X^{0}\right)$ with respect to this system, the following holds:

$$
\text { (i) } X^{*}\left(X^{0}\right)=0 \text {; }
$$

(ii) $\perp_{0} \mathcal{S}=\left\{X \in \mathbb{R}^{n} \mid X_{1}=\cdots=X_{n-1}=0\right\}$;

(iii) $X^{0} \equiv\left(0, \ldots, 0, X_{n}^{0}\right), d\left(X^{0}\right)=X_{n}^{0}$;

$$
\text { (iv) } \frac{\partial^{2} p_{n}}{\partial X_{i} \partial X_{j}}(0)=\frac{\partial^{2} p_{n}}{\partial X_{i}^{2}}(0) \delta_{i j} \quad(i, j=1, \ldots, n-1) \text {. }
$$

Lemma 2.5 Let assumption $\left(H_{1}\right)$ be satisfied. There exist $\varepsilon_{0} \in(0, \sigma)$ and $C_{0}>0$ such that, if $\varepsilon \in\left(0, \varepsilon_{0}\right), x^{0} \in \mathcal{S}^{\varepsilon}$ is fixed and the choice $(C)$ is made, then:

(i) for any $i=1, \ldots, n$,

$$
\left.\frac{\partial d(X)}{\partial X_{i}}\right|_{X=X^{0}}=\delta_{i n}
$$

(ii) we have

$$
\begin{aligned}
\left|\frac{\partial^{2} d(X)}{\partial X_{i} \partial X_{j}}\right|_{X=X^{0}} \mid \leqslant C_{0} & \text { if } i=j=1, \ldots, n-1, \\
\left.\frac{\partial^{2} d(X)}{\partial X_{i} \partial X_{j}}\right|_{X=X^{0}}=0 & \text { otherwise. }
\end{aligned}
$$

We refer the reader to [MP], [PPT] for the proof of the above lemma.

\section{Proof of Theorem 1.1 and Corollary 1.2}

We adapt to the present situation the proof of Theorem 1 in [KK]. In the proof of Theorem $1.1 \mathrm{a}$ central role will be played by

LEMmA 3.1 Let assumptions $\left(H_{1}\right)-\left(H_{2}\right)$ be satisfied. Let $u$ be a solution to problem (1.1), and $T>0$. Suppose that $\operatorname{supp} u(\cdot, t) \cap \mathcal{S}=\emptyset$ for any $\tau \in(0, T)$. Then for any $\tau \in(0, T)$,

$$
\int_{\Omega} \rho(x) u(x, \tau) \mathrm{d} x=\int_{\Omega} \rho(x) u_{0}(x) \mathrm{d} x .
$$


Proof. Let $\tau \in(0, T)$; take $\varepsilon>0$ so small that

$$
u(x, t)=0 \quad \text { for any }(x, t) \in \overline{\mathcal{S}^{\varepsilon}} \times(0, \tau) .
$$

Let $\eta \in C^{\infty}([0, \tau]), 0 \leqslant \eta \leqslant 1$,

$$
\eta(t)=\left\{\begin{array}{ll}
1 & \text { if } t \in[0, \tau-2 \delta] \\
0 & \text { if } t \in[\tau-\delta, \tau]
\end{array} \quad(\delta>0)\right.
$$

and $\chi \in C_{0}^{\infty}\left(\Omega \backslash \overline{\mathcal{S}^{\varepsilon / 2}}\right), \chi \equiv 1$ in $\bar{\Omega} \backslash \overline{\mathcal{S}^{\varepsilon}}$. Equality (2.1) with $\psi=\eta \chi, \Omega_{1}=\Omega \backslash \overline{\mathcal{S}^{\varepsilon / 2}}$ gives

$$
\begin{aligned}
\int_{0}^{\tau} \int_{\Omega \backslash \overline{\mathcal{S}^{\varepsilon / 2}}}\left\{\rho u \partial_{t} \psi+G(u) \Delta \psi\right\} \mathrm{d} x \mathrm{~d} t= & \int_{\Omega \backslash \overline{\mathcal{S}^{\varepsilon / 2}}} \rho\left[u(x, \tau) \psi(x, \tau)-u_{0}(x) \psi(x, 0)\right] \mathrm{d} x \\
& +\int_{0}^{\tau} \int_{\mathcal{A}^{\varepsilon / 2}} G(u)\langle\nabla \psi, \nu\rangle \mathrm{d} \sigma \mathrm{d} t,
\end{aligned}
$$

hence

$$
\int_{0}^{\tau} \int_{\Omega \backslash \overline{\mathcal{S}^{\varepsilon}}} \rho u \partial_{t} \psi \mathrm{d} x \mathrm{~d} t=-\int_{\Omega \backslash \overline{\mathcal{S}^{\varepsilon}}} \rho u_{0} \mathrm{~d} x .
$$

It is easily seen that (3.3) when $\delta \rightarrow 0^{+}$yields

$$
-\int_{\Omega \backslash \overline{\mathcal{S}^{\varepsilon}}} \rho u(x, \tau) \mathrm{d} x \mathrm{~d} t=-\int_{\Omega \backslash \overline{\mathcal{S}^{\varepsilon}}} \rho u_{0} \mathrm{~d} x .
$$

Letting $\varepsilon \rightarrow 0^{+}$, by the monotone convergence theorem we get the conclusion.

Lemma 3.2 Let the assumptions of Theorem 1.1 be satisfied. Then there exists a minimal positive solution $W$ to the equation

$$
\Delta U=-\rho u_{0} \quad \text { in } \Omega .
$$

Moreover, $W \in C(\bar{\Omega})$ and $W=0$ on $\mathcal{S}$.

Proof. For any $\varepsilon>0$ let $W_{\varepsilon}$ be the solution to the problem

$$
\begin{cases}\Delta U=-\rho u_{0} & \text { in } \Omega \backslash \overline{\mathcal{S}^{\varepsilon}} \\ U=0 & \text { in } \mathcal{A}^{\varepsilon} .\end{cases}
$$

By the strong maximum principle we have, for any $\varepsilon>0$,

$$
W_{\varepsilon}>0 \quad \text { in } \Omega \backslash \mathcal{S}^{\varepsilon} .
$$

This implies that if $\varepsilon_{1} \geqslant \varepsilon_{2}>0$, then $W_{\varepsilon_{1}}$ is a solution, while $W_{\varepsilon_{2}}$ is a supersolution, to the problem

$$
\begin{cases}\Delta U=-\rho u_{0} & \text { in } \Omega \backslash \overline{\mathcal{S}^{\varepsilon_{1}}}, \\ U=0 & \text { in } \mathcal{A}^{\varepsilon_{1}} .\end{cases}
$$

Thus, again by the maximum principle, we infer that for any $\varepsilon_{1} \geqslant \varepsilon_{2}>0$,

$$
W_{\varepsilon_{1}} \leqslant W_{\varepsilon_{2}} \text { in } \Omega \backslash \mathcal{S}^{\varepsilon_{1}} .
$$


Observe that, since supp $u_{0} \cap \mathcal{S}=\emptyset$ and $\rho \in C(\Omega)$, there exists (see e.g. [PPT]) a supersolution $V \in C^{2}\left(\mathcal{S}^{\varepsilon}\right) \cap C(\bar{\Omega})$ (for $\varepsilon>0$ sufficiently small) to equation (3.4) such that $V>0$ in $\Omega$ and

$$
V=0 \quad \text { on } \mathcal{S} \text {. }
$$

In particular, $V$ is a supersolution to problem (3.5).

Thus by comparison principles we have

$$
W_{\varepsilon} \leqslant V \quad \text { in } \Omega \backslash \mathcal{S}^{\varepsilon} .
$$

By usual compactness arguments, there exists a subsequence $\left\{W_{\varepsilon_{m}}\right\} \subseteq\left\{W_{\varepsilon}\right\}$ which converges uniformly on compact subsets of $\Omega$. Let

$$
W:=\lim _{m \rightarrow+\infty} W_{\varepsilon_{m}} \quad \text { in } \Omega .
$$

Then $W$ is a solution to equation (3.4). Moreover, from (3.6)-(3.7) and (3.9) if follows that

$$
0<W \leqslant V \quad \text { in } \Omega .
$$

By (3.10) we see that $W \in C(\bar{\Omega})$ and $W=0$ on $\mathcal{S}$. Clearly, $W$ is minimal among all positive solutions to equation (3.4).

Lemma 3.3 Let the assumptions of Theorem 1.1 be satisfied. Then there exists a minimal solution $\underline{u}$ to problem (1.1); moreover, for any $t>0$,

$$
\int_{0}^{t} G(\underline{u}(x, \tau)) \mathrm{d} \tau \leqslant W(x) \quad(x \in \Omega),
$$

$W$ being the minimal positive solution to equation (3.4) defined in Lemma 3.2.

Proof. For any $\varepsilon>0$ let $u_{\varepsilon}$ be the unique solution to problem (2.2). By comparison results we have

$$
0 \leqslant u_{\varepsilon} \leqslant\left\|u_{0}\right\|_{\infty} \quad \text { in } Q_{\varepsilon} .
$$

By usual compactness arguments, there exists a subsequence $\left\{u_{\varepsilon_{m}}\right\} \subseteq\left\{u_{\varepsilon}\right\}$ which converges uniformly on compact subsets of $\Omega \times(0,+\infty)$ to a solution $\underline{u}$ to problem (1.1). Clearly, $\underline{u}$ is the minimal nonnegative solution to problem (1.1).

Define

$$
\begin{array}{cl}
\underline{U}(x, t):=\int_{0}^{t} G(\underline{u}(x, \tau)) \mathrm{d} \tau & ((x, t) \in Q), \\
U_{\varepsilon}(x, t):=\int_{0}^{t} G\left(u_{\varepsilon}(x, \tau)\right) \mathrm{d} \tau & \left((x, t) \in Q_{\varepsilon}\right) .
\end{array}
$$

Observe that $U_{\varepsilon_{m}} \rightarrow \underline{U}$ in $\Omega \times(0,+\infty)$ as $m \rightarrow+\infty$.

It is straightforward to show that for any $t>0$ the function $U_{\varepsilon}(\cdot, t)$ is a subsolution to problem (3.5). In fact, by Definition 2.2 we obtain

$$
\begin{aligned}
\int_{\Omega_{1}} U_{\varepsilon}(x, \tau) \Delta \psi(x) \mathrm{d} x & =\int_{\Omega_{1}} \rho(x)\left[u_{\varepsilon}(x, \tau)-u_{0}(x)\right] \psi(x) \mathrm{d} x+\int_{\partial \Omega_{1} \backslash \mathcal{A}^{\varepsilon}} U_{\varepsilon}(x, \tau)\langle\nabla \psi(x), \nu\rangle \mathrm{d} \sigma \\
& \geqslant-\int_{\Omega_{1}} \rho(x) u_{0}(x) \psi(x) \mathrm{d} x+\int_{\partial \Omega_{1} \backslash \mathcal{A}^{\varepsilon}} U_{\varepsilon}(x, \tau)\langle\nabla \psi(x), \nu\rangle \mathrm{d} \sigma
\end{aligned}
$$

for any $\Omega_{1}$ and $\psi=\psi(x)$ as in Definition 2.4 and $\tau>0$. 
Let $\left\{W_{\varepsilon_{m}}\right\}$ be the sequence of solutions to problems (3.5) with $\varepsilon=\varepsilon_{m}$ introduced in the proof of Lemma 3.2. By comparison results we obtain

$$
W_{\varepsilon_{m}} \geqslant U_{\varepsilon_{m}} \quad \text { in } \Omega \backslash S^{\varepsilon_{m}}
$$

letting $m \rightarrow+\infty$ we get (3.11).

Proof of Theorem 1.1. Let $\underline{u}$ be the minimal solution to problem (1.1). From (3.11) we have

$$
\underline{U}(x, t) \leqslant \max _{\bar{\Omega}} W=: C_{1} \quad \text { for any }(x, t) \in Q ;
$$

here $\underline{U}$ is given by (3.12). Therefore

$$
\int_{0}^{t} \int_{\Omega \backslash \overline{\mathcal{S}^{\varepsilon}}} G(\underline{u}(x, \tau)) \mathrm{d} x \mathrm{~d} \tau \leqslant C_{1} \operatorname{meas}(\Omega) .
$$

From (3.15) we obtain

$$
\int_{t}^{t+1} G(\underline{u}(x, \tau)) \mathrm{d} x \mathrm{~d} \tau \rightarrow 0 \quad \text { as } t \rightarrow+\infty .
$$

Fix $\sigma>0$ arbitrarily. Since $\rho \in L^{1}(\Omega)$, we can find $\bar{\varepsilon}=\bar{\varepsilon}(\sigma)>0$ such that

$$
\int_{\mathcal{S}_{\bar{\varepsilon}}^{\bar{\varepsilon}}} \rho(x) \mathrm{d} x<\sigma ;
$$

moreover, by $\left(H_{3}\right)$ we have

$$
\underline{u} \leqslant \frac{\sigma}{\operatorname{meas}\left(\Omega \backslash \mathcal{S}^{\bar{\varepsilon}}\right)}+\bar{a} G(\underline{u}) \quad \text { in } Q
$$

for some $\bar{a}=\bar{a}(\sigma)>0$.

Using (3.16) and (3.18) we have

$$
\begin{aligned}
\int_{t}^{t+1} \int_{\Omega \backslash \mathcal{S}^{\bar{\varepsilon}}} \rho \underline{u} \mathrm{~d} x \mathrm{~d} \tau & \leqslant \sup _{\Omega \backslash \mathcal{S}^{\bar{\varepsilon}}} \rho \int_{t}^{t+1} \int_{\Omega \backslash \mathcal{S}^{\bar{\varepsilon}}} \underline{u} \mathrm{~d} x \mathrm{~d} t \\
& \leqslant \sup _{\Omega \backslash \mathcal{S}^{\bar{\varepsilon}}} \rho\left[\sigma+\bar{a} \int_{t}^{t+1} \int_{\Omega \backslash \mathcal{S}^{\bar{\varepsilon}}} G(\underline{u}) \mathrm{d} x \mathrm{~d} \tau\right] \leqslant \sup _{\Omega \backslash \mathcal{S}^{\bar{\varepsilon}}} \rho(1+\bar{a}) \sigma
\end{aligned}
$$

for any $t \geqslant \bar{t}$ for some $\bar{t}=\bar{t}(\sigma)>0$. Inequalities (3.17) and (3.19) give

$$
\begin{aligned}
\int_{t}^{t+1} \int_{\Omega} \rho \underline{u} \mathrm{~d} x \mathrm{~d} \tau & =\int_{t}^{t+1} \int_{\Omega \backslash \mathcal{S}^{\bar{\varepsilon}}} \rho \underline{u} \mathrm{~d} x \mathrm{~d} \tau+\int_{t}^{t+1} \int_{\mathcal{S}_{\bar{\varepsilon}}} \rho \underline{u} \mathrm{~d} x \mathrm{~d} \tau \\
& \leqslant \sup _{\Omega \backslash \mathcal{S}^{\bar{\varepsilon}}} \rho(1+\bar{a}) \sigma+\left\|u_{0}\right\|_{\infty} \sigma=\left(\sup _{\Omega \backslash \mathcal{S}^{\bar{\varepsilon}}} \rho(1+\bar{a})+\left\|u_{0}\right\|_{\infty}\right) \sigma \quad \text { for any } t \geqslant \bar{t} .
\end{aligned}
$$

Hence equality (3.1) cannot be satisfied with $u=\underline{u}$. Then Lemma 3.2 implies the result. 
LEMmA 3.4 Let the assumptions of Corollary 1.2 be satisfied; let $\alpha \in(0,1)$. Then the minimal solution to problem (1.1) satisfies

$$
\int_{0}^{t} G(\underline{u}(x, \tau)) \mathrm{d} \tau \leqslant C[d(x)]^{\alpha} \quad \text { for any } x \in \mathcal{S}^{\tilde{\varepsilon}}
$$

for some $C>0, \tilde{\varepsilon}>0$.

In order to prove Lemma 3.4 we will use problems of the following type:

$$
\begin{cases}\Delta U=0 & \text { in } \mathcal{S}^{\tilde{\varepsilon}} \backslash \overline{\mathcal{S}^{\varepsilon}}, \\ U=0 & \text { in } \mathcal{A}^{\varepsilon}, \\ U=\gamma & \text { in } \mathcal{A}^{\tilde{\varepsilon}}\end{cases}
$$

here $\tilde{\varepsilon}>\varepsilon>0$ and $\gamma \in C\left(\mathcal{A}^{\tilde{\varepsilon}}\right)$.

DEFinition 3.5 By a supersolution of problem (3.21) we mean a function $U \in C\left(\overline{\mathcal{S}^{\tilde{\varepsilon}} \backslash \mathcal{S}^{\varepsilon}}\right)$ such that

$$
\int_{\Omega_{1}} U \Delta \psi \mathrm{d} x \leqslant \int_{\partial \Omega_{1} \backslash \mathcal{A}^{\varepsilon}} U\langle\nabla \psi, \nu\rangle \mathrm{d} \sigma+\int_{\partial \Omega_{1} \cap \mathcal{A}^{\tilde{\varepsilon}}} \gamma\langle\nabla \psi, \nu\rangle \mathrm{d} \sigma
$$

for any open set $\Omega_{1} \subseteq \overline{\mathcal{S}^{\tilde{\varepsilon}} \backslash \mathcal{S}^{\varepsilon}}$ with smooth boundary, $\psi \in C^{2}\left(\overline{\Omega_{1}}\right), \psi \geqslant 0, \psi=0$ in $\partial \Omega_{1}$; here $v$ denotes the outer normal to $\Omega_{1}$. Solutions and subsolutions are defined accordingly.

Proof of Lemma 3.4. Define

$$
V(x):=C[d(x)]^{\alpha} \quad\left(x \in \mathcal{S}^{\varepsilon_{1}}\right)
$$

with $C>0, \varepsilon_{1} \in\left(0, \varepsilon_{0}\right)$ to be chosen later. For any $x \in \mathcal{S}^{\varepsilon_{1}}$ and $i, j=1, \ldots, n$ we have

$$
\begin{aligned}
\frac{\partial V(x)}{\partial x_{i}} & =C \alpha[d(x)]^{\alpha-1} \frac{\partial d(x)}{\partial x_{i}}, \\
\frac{\partial^{2} V(x)}{\partial x_{i} \partial x_{j}} & =C \alpha\left\{(\alpha-1)[d(x)]^{\alpha-2} \frac{\partial d(x)}{\partial x_{j}} \frac{\partial d(x)}{\partial x_{i}}+[d(x)]^{\alpha-1} \frac{\partial^{2} d(x)}{\partial x_{i} \partial x_{j}}\right\} .
\end{aligned}
$$

From (2.9)-(2.10) and (3.22) we get

$$
\Delta V(x) \leqslant C \alpha[d(x)]^{\alpha-2}\left\{\alpha-1+(n-1) C_{0} d(x)\right\} \leqslant 0 \quad\left(x \in \mathcal{S}^{\varepsilon_{1}}\right),
$$

taking $\varepsilon_{1} \in\left(0, \varepsilon_{0}\right)$ small enough. Choose $\tilde{\varepsilon} \in\left(0, \varepsilon_{1}\right)$ so small that

$$
\operatorname{supp} u_{0} \subseteq \Omega \backslash \mathcal{S}^{2 \tilde{\varepsilon}} ;
$$

then fix $m_{0} \in \mathbb{N}$ such that $\varepsilon_{m} \in(0, \tilde{\varepsilon})$ for any $m>m_{0}$.

Consider the sequences of functions $\left\{u_{\varepsilon_{m}}\right\},\left\{U_{\varepsilon_{m}}\right\}$ introduced in the proof of Lemma 3.3. It is easily seen that for any $t>0$ the function $U_{\varepsilon_{m}}(\cdot, t)$ is a subsolution to the problem

$$
\left\{\begin{array}{ll}
\Delta U=0 & \text { in } \mathcal{S}^{\tilde{\varepsilon}} \backslash \overline{\mathcal{S}^{\varepsilon_{m}}} \\
U=0 & \text { in } \mathcal{A}^{\varepsilon_{m}}
\end{array} \quad\left(m>m_{0}\right) .\right.
$$


In fact, by Definition 2.2 using (3.13), (3.14), (3.24) we obtain

$$
\begin{aligned}
\int_{\Omega_{1}} U_{\varepsilon_{m}}(x, \tau) \Delta \psi(x) \mathrm{d} x \geqslant & -\int_{\Omega_{1}} \rho(x) u_{0} \psi(x) \mathrm{d} x+\int_{\partial \Omega_{1} \backslash \mathcal{A}^{\varepsilon}} U_{\varepsilon_{m}}(x, \tau)\langle\nabla \psi(x), \nu\rangle \mathrm{d} \sigma \\
= & \int_{\partial \Omega_{1} \backslash\left(\mathcal{A}^{\varepsilon} \cup \mathcal{A}^{\tilde{\varepsilon}}\right)} U_{\varepsilon}(x, \tau)\langle\nabla \psi(x), \nu\rangle \mathrm{d} \sigma \\
& +\int_{\partial \Omega_{1} \cap \mathcal{A}^{\tilde{\varepsilon}}} U_{\varepsilon_{m}}(x, \tau)\langle\nabla \psi(x), \nu\rangle \mathrm{d} \sigma \\
\geqslant & \int_{\partial \Omega_{1} \backslash\left(\mathcal{A}^{\varepsilon} \cup \mathcal{A}^{\tilde{\varepsilon}}\right)} U_{\varepsilon_{m}}(x, \tau)\langle\nabla \psi(x), \nu\rangle \mathrm{d} \sigma+\int_{\partial \Omega_{1} \cap \mathcal{A}^{\tilde{\varepsilon}}} C_{1}\langle\nabla \psi(x), \nu\rangle \mathrm{d} \sigma
\end{aligned}
$$

for any $\Omega_{1}$ and $\psi=\psi(x)$ as in Definition 3.5, $\tau>0, m>m_{0}$; here $C_{1}>0$ is the constant defined in (3.14), and the inequality

$$
\langle\nabla \psi, v\rangle \leqslant 0 \quad \text { on } \partial \Omega_{1}
$$

has been used.

Set $C:=C_{1} / \tilde{\varepsilon}^{\alpha}$. Then, in view of (3.23), $V$ is a supersolution (in the classical sense) to problem (3.25). By comparison results we have

$$
U_{\varepsilon_{m}}(x) \leqslant V(x) \quad\left(x \in \mathcal{S}^{\tilde{\varepsilon}} \backslash \mathcal{S}^{\varepsilon_{m}}\right) .
$$

Letting $m \rightarrow+\infty$ yields the result.

Proof of Corollary 1.2. Let $\underline{u}$ be the minimal positive solution to problem (1.1) introduced in Lemma 3.3. Fix any $\sigma>0$. In view of Lemma 3.4, since $\rho[d(x)]^{\alpha / m} \in L^{1}(\Omega)$ we obtain, for any $t>0$,

$$
\begin{aligned}
\int_{t}^{t+1} \int_{\mathcal{S}^{\varepsilon}} u \rho \mathrm{d} x \mathrm{~d} \tau & \leqslant \int_{\mathcal{S}^{\varepsilon}} \rho\left[\int_{t}^{t+1} u \mathrm{~d} \tau\right] \mathrm{d} x \leqslant \int_{\mathcal{S}^{\varepsilon}} \rho\left[\int_{t}^{t+1} u^{m} \mathrm{~d} \tau\right]^{1 / m} \mathrm{~d} x \\
& \leqslant C^{1 / m} \int_{\mathcal{S}^{\varepsilon}} \rho(x)[d(x)]^{\alpha / m} \mathrm{~d} x<\sigma,
\end{aligned}
$$

choosing $\varepsilon>0$ small enough. Repeating the proof of Theorem 1.1, using inequality (3.27) instead of (3.17), yields the conclusion.

\section{Proof of Theorem 1.3}

Proof of Theorem 1.3. (i) Let $\alpha>2$. Define

$$
\tilde{v}(x, t):=\left[a-\frac{1}{d(x)(b t+1)^{\beta}}\right]_{+}^{1 /(m-1)} \equiv F^{1 /(m-1)} \quad\left((x, t) \in \mathcal{S}^{\hat{\varepsilon}} \times[0,+\infty)\right),
$$

where $a>0, b>0, \beta>1$ are constants to be fixed. Take $\hat{\varepsilon} \in(0, \sigma)$ such that supp $u_{0} \subseteq \Omega \backslash \overline{\mathcal{S}^{\hat{\varepsilon}}}$.

Let $\mathcal{N}:=\left\{(x, t) \in \mathcal{S}^{\hat{\varepsilon}} \times[0,+\infty) \mid \tilde{v}(x, t)=0\right\}$. Choose

$$
a \geqslant 3 / \hat{\varepsilon}+\left\|u_{0}\right\|_{\infty}^{m-1}
$$


thus

$$
d(x)<\hat{\varepsilon} / 2 \quad \text { for any }(x, t) \in \mathcal{N} .
$$

For any $(x, t) \in\left[\mathcal{S}^{\hat{\varepsilon}} \times(0,+\infty)\right] \backslash \mathcal{N}=: \mathcal{D}$ we have:

$$
\begin{aligned}
\frac{\partial \tilde{v}(x, t)}{\partial t}= & \frac{\beta b}{m-1}[d(x)]^{-1}(b t+1)^{-\beta-1} F^{-1+1 /(m-1)}, \\
\frac{\partial\left\{[\tilde{v}(x, t)]^{m}\right\}}{\partial x_{i}}= & \frac{m}{m-1} F^{1 /(m-1)}(b t+1)^{-\beta}[d(x)]^{-2} \frac{\partial d(x)}{\partial x_{i}} \\
\frac{\partial^{2}\left\{[\tilde{v}(x, t)]^{m}\right\}}{\partial x_{i} \partial x_{j}}= & \frac{m}{m-1}(b t+1)^{-\beta}\left\{\frac{F^{-1+1 /(m-1)}}{m-1}(b t+1)^{-\beta}[d(x)]^{-4} \frac{\partial d(x)}{\partial x_{i}} \frac{\partial d(x)}{\partial x_{j}}\right. \\
& \left.+F^{1 /(m-1)}\left[-2[d(x)]^{-3} \frac{d(x)}{\partial x_{i}} \frac{d(x)}{\partial x_{j}}+[d(x)]^{-2} \frac{\partial^{2} d(x)}{\partial x_{i} \partial x_{j}}\right]\right\} ;
\end{aligned}
$$

here $i, j=1, \ldots, n$. From (2.6), (2.7), (4.4), (4.6), we obtain

$$
\begin{aligned}
& \rho(x) \frac{\partial \tilde{v}}{\partial t}-\Delta\left\{[\tilde{v}(x, t)]^{m}\right\} \\
& \geqslant \frac{[d(x)]^{-1}}{m-1}(b t+1)^{-\beta-1} F^{-1+1 /(m-1)}\left\{b \beta \rho(x)-\frac{m}{m-1}(b t+1)^{-\beta+1}[d(x)]^{-3}\right\} \\
& \quad \text { for any }(x, t) \in \mathcal{D} .
\end{aligned}
$$

By (4.7) and (1.5), if $\alpha \geqslant 3, \beta>1, b \geqslant \frac{m}{C \beta(m-1)}$, then

$$
\rho \partial_{t} \tilde{v}-\Delta \tilde{v}^{m} \geqslant 0 \quad \text { in } \mathcal{D} \text {. }
$$

Now suppose $\alpha \in(2,3)$. Define $\gamma:=\frac{1}{3-\alpha}$ and take $\beta \geqslant \frac{\gamma}{\gamma-1}=\frac{1}{\alpha-2}$. Observe that

$$
[d(x)]^{-1 / \gamma}<a^{1 / \gamma}(b t+1)^{\beta / \gamma} .
$$

Inequalities (4.7) and (4.9) yield, for any $(x, t) \in \mathcal{D}$,

$$
\begin{aligned}
\rho(x) \partial_{t} \tilde{v}(x, t) & -\Delta\left\{[\tilde{v}(x, t)]^{m}\right\} \\
\geqslant & \frac{[d(x)]^{-1}}{m-1}(b t+1)^{-\beta-1} F^{-1+1 /(m-1)}\left\{\beta b \rho(x)-\frac{m(b t+1)^{-\beta+1}}{m-1}[d(x)]^{-1 / \gamma-3+1 / \gamma}\right\} \\
\geqslant & \frac{[d(x)]^{-1}}{m-1}(b t+1)^{-\beta-1} F^{-1+1 /(m-1)} \\
& \cdot\left\{\beta b[d(x)]^{-3+1 / \gamma}-\frac{m}{m-1}(b t+1)^{-\beta+1+\beta / \gamma} a^{1 / \gamma}[d(x)]^{-3+1 / \gamma}\right\} \geqslant 0,
\end{aligned}
$$

taking $b \geqslant \frac{m}{C \beta(m-1)} a^{1 / \gamma}$, for $1-\beta+\beta / \gamma \leqslant 0$. Furthermore, observe that

$$
\nabla\left(\tilde{v}^{m}\right)=0 \quad \text { in } \partial \mathcal{N} \cap Q
$$

Define

$$
\hat{v}(x, t):=\left[a-\frac{1}{\hat{\varepsilon}(b t+1)^{\beta}}\right]^{1 /(m-1)} \quad\left((x, t) \in\left(\Omega \backslash \mathcal{S}^{\hat{\varepsilon}}\right) \times[0,+\infty)\right)
$$


(notice that, due to (4.2), $a-\frac{1}{\hat{\varepsilon}(b t+1)^{\beta}}>0$ ). Clearly,

$$
\rho \partial_{t} \hat{v}-\Delta\left(\hat{v}^{m}\right) \geqslant 0 \quad \text { in }(\Omega \backslash \overline{\mathcal{S} \hat{\varepsilon}}) \times(0,+\infty) .
$$

From (4.5) we have

$$
\left\langle\nabla\left(\tilde{v}^{m}\right), v_{\hat{\varepsilon}}\right\rangle=\left\langle\nabla\left(\tilde{v}^{m}\right), \nabla d(x)\right\rangle \geqslant\left\langle\nabla\left(\hat{v}^{m}\right), v_{\hat{\varepsilon}}\right\rangle=0 \quad \text { in } \mathcal{A}^{\hat{\varepsilon}} \times(0,+\infty),
$$

where $\nu_{\hat{\varepsilon}}$ is the outer normal to $\mathcal{S}^{\hat{\varepsilon}}$ at $\mathcal{A}^{\hat{\varepsilon}}$.

Define

$$
v(x, t):= \begin{cases}\tilde{v}(x, t) & \text { if }(x, t) \in \mathcal{S}^{\hat{\varepsilon}} \times[0,+\infty), \\ \hat{v}(x, t) & \text { if }\left(\Omega \backslash \mathcal{S}^{\hat{\varepsilon}}\right) \times[0,+\infty) .\end{cases}
$$

In view of (4.2)-(4.3), we have $v \in C(\Omega \times[0,+\infty))$ and

$$
v(x, 0) \geqslant u_{0}(x) \quad(x \in \Omega) .
$$

We claim that $v$ is a supersolution to problem (1.1). In fact, take $\tau>0, \Omega_{1}$ and $\psi$ as in Definition 2.1. By (4.8), (4.10), (4.13) we have

$$
\int_{0}^{\tau} \int_{\Omega_{1}}\left\{\rho \partial_{t} v-\Delta\left(v^{m}\right)\right\} \psi \mathrm{d} x \mathrm{~d} t \geqslant 0 .
$$

Hence, due to (4.1) we get

$$
\begin{aligned}
-\int_{0}^{\tau} \int_{\Omega_{1}} \rho v \partial_{t} \psi \mathrm{d} x \mathrm{~d} t+ & \int_{\Omega_{1}} \rho[v(x, t) \psi(x, t)-v(x, 0) \psi(x, 0)] \mathrm{d} x \\
& -\int_{0}^{\tau} \int_{\Omega_{1} \backslash \mathcal{S}^{\hat{\varepsilon}}} \Delta\left(\hat{v}^{m}\right) \psi \mathrm{d} x \mathrm{~d} t-\int_{0}^{\tau} \int_{\Omega_{1} \cap \mathcal{S}^{\hat{\varepsilon}}} \Delta\left(\tilde{v}^{m}\right) \psi \mathrm{d} x \mathrm{~d} t \geqslant 0 .
\end{aligned}
$$

Then, integrating by parts, using (4.1), (4.3) and (4.11) we obtain

$$
\begin{aligned}
-\int_{0}^{\tau} & \int_{\Omega_{1}} \rho v \partial_{t} \psi \mathrm{d} x \mathrm{~d} t+\int_{\Omega_{1}} \rho[v(x, t) \psi(x, t)-v(x, 0) \psi(x, 0)] \mathrm{d} x \\
& -\int_{0}^{\tau} \int_{\Omega_{1} \backslash \mathcal{S}_{\hat{\varepsilon}}} \hat{v}^{m} \Delta \psi \mathrm{d} x \mathrm{~d} t+\int_{0}^{\tau} \int_{\partial \Omega_{1} \backslash \mathcal{S}_{\hat{\varepsilon}}} \hat{v}^{m}\langle\nabla \psi, v\rangle \mathrm{d} \sigma \mathrm{d} t-\int_{0}^{\tau} \int_{\mathcal{A}^{\hat{\varepsilon}} \cap \Omega_{1}} \hat{v}^{m}\left\langle\nabla \psi, v_{\hat{\varepsilon}}\right\rangle \mathrm{d} \sigma \mathrm{d} t \\
& -\int_{0}^{\tau} \int_{\Omega_{1} \cap \mathcal{S}^{\hat{\varepsilon}}} \tilde{v}^{m} \Delta \psi \mathrm{d} x \mathrm{~d} t-\int_{0}^{\tau} \int_{\mathcal{A}_{\hat{\varepsilon}} \cap \Omega_{1}}\left\langle\nabla\left(\tilde{v}^{m}\right), v_{\hat{\varepsilon}}\right\rangle \psi \mathrm{d} \sigma \mathrm{d} t \\
& +\int_{0}^{\tau} \int_{\mathcal{A}_{\hat{\varepsilon} \cap \Omega_{1}}} \tilde{v}^{m}\left\langle\nabla \psi, v_{\hat{\varepsilon}}\right\rangle \mathrm{d} \sigma \mathrm{d} t+\int_{0}^{\tau} \int_{\partial \Omega_{1} \cap \mathcal{S}^{\hat{\varepsilon}}} \tilde{v}^{m}\langle\nabla \psi, v\rangle \mathrm{d} \sigma \mathrm{d} t \geqslant 0 .
\end{aligned}
$$

Therefore, from (4.14), (4.16), (3.26), since $v \in C(Q)$, we get

$$
\begin{aligned}
\int_{0}^{\tau} \int_{\Omega_{1}}\left\{\rho v \partial_{t} \psi+v^{m} \Delta \psi\right\} \mathrm{d} x \mathrm{~d} t \leqslant & \int_{\Omega_{1}} \rho\left[v(x, \tau) \psi(x, \tau)-u_{0}(x) \psi(x, 0)\right] \mathrm{d} x \\
& +\int_{0}^{\tau} \int_{\partial \Omega_{1}} v^{m}\langle\nabla \psi, v\rangle \mathrm{d} \sigma \mathrm{d} t .
\end{aligned}
$$

Hence the claim has been proved. 
Now, let $u$ be any solution to problem (1.1). Let $\underline{u}$ denote the minimal positive solution to problem (1.1) considered in Lemma 3.3, and for any $\varepsilon>0$ let $u_{\varepsilon}$ denote the solution to problem (2.2). By uniqueness results (see [P2]) we deduce that

$$
u \equiv \underline{u} \quad \text { in } Q .
$$

Observe that the claim above implies that $v$ is a supersolution to problem (2.2) for any $\varepsilon>0$. Hence by comparison principles, for any $\varepsilon>0$,

$$
u_{\varepsilon} \leqslant v \quad \text { in } Q_{\varepsilon} .
$$

This implies that $\underline{u} \leqslant v$ in $Q$; thus by (4.18) we deduce

$$
u \leqslant v \quad \text { in } Q .
$$

Since supp $v(\cdot, t)$ is compact for any $t>0$, by (4.20), also $\operatorname{supp} u(\cdot, t)$ is compact for any $t>0$. Moreover, (4.20), (4.12), (4.15) and (4.1) imply (1.6).

(ii) Let $\alpha=2$. The result follows by arguing as in (i) above, replacing the definition of $\tilde{v}$ in (4.1) by the following:

$$
\tilde{v}(x, t):=\left[a-\frac{1}{d(x) e^{\beta t}}\right]_{+} \equiv F^{1 /(m-1)} \quad\left((x, t) \in \mathcal{S}^{\hat{\varepsilon}} \times[0,+\infty)\right),
$$

where $a>0, b>0, \beta>1$ are constants to be chosen appropriately.

REMARK 4.1 Observe that when $\Omega$ is a bounded interval of $\mathbb{R}$ or, for instance, $\Omega=B_{R}\left(x_{0}\right) \subseteq \mathbb{R}^{n}$ $(n \geqslant 1)$ for some $x_{0} \in \mathbb{R}^{n}$ and $R>0$, then the distance $d(x)(x \in \Omega)$ can be explicitly computed, hence the proofs of Lemma 3.4 and Theorem 1.3 become more direct.

\section{REFERENCES}

[AD] Ambrosio, L., \& DAnCER, N. Calculus of Variations and Partial Differential Equations: Topics on Geometrical Evolution Problem and Degree Theory. Springer (2000). MR 1757706

[A] ARnold, V. I. Lectures on Partial Differential Equations. Springer (2004). Zbl 1076.35002 MR 2031206

[EK] EIdus, D., \& KAMIN, S. The filtration equation in a class of functions decreasing at infinity. Proc. Amer. Math. Soc. 120 (1994), 825-830. Zbl 0791.35065 MR 1169025

[F] Foote, R. L. Regularity of distance function. Proc. Amer. Math. Soc. 92 (1984), 153-155. Zbl 0528.53005 MR 0749908

[GHP] Guedda, M., Hilhorst, D., \& Peletier, M. Disappearing interfaces in nonlinear diffusion. $A d v$. Math. Sci. Appl. 7 (1997), 695-710. Zbl 0891.35071 MR 1476273

[KK] Kamin, S., \& Kersner, R. Disappearance of interfaces in finite time. Meccanica 28 (1993), 117120. Zbl 0786.76088

[KKT] KAmin, S., Kersner, R., \& TESEI, A. On the Cauchy problem for a class of parabolic equations with variable density. Rend. Lincei Mat. Appl. 9 (1998), 279-298. Zbl 0926.35045 MR 1722787

[KT] Kersner, R., \& TESEI, A. Well-posedness of initial value problems for singular parabolic equations. J. Differential Equations 199 (2004), 47-76. Z Zbl 1061.35030 MR 2041511

[MP] Matano, H., \& Pozio, M. A. Rich dynamical structure of some nonlinear degenerate diffusion equations. Preprint no. 8 of Dip. di Matematica, Università "La Sapienza", Roma (1997). 
[PPT] Pozio, M. A., Punzo, F., \& TeseI, A. Criteria for well-posedness of degenerate elliptic and parabolic problems. J. Math. Pures Appl. 90 (2008), 353-386. Zbl 1171.35056 MR 2454711

[PT] PozIo, M. A., \& TESEI, A. On the uniqueness of bounded solutions to singular parabolic problems. Discrete Contin. Dynam. Systems 13 (2005), 117-137. Zbl 1165.35403 MR 2128795

[P1] Punzo, F. On the Cauchy problem for nonlinear parabolic equations with variable density. J. Evol. Equations 9 (2009), 429-447. MR 2529731

[P2] PUnzo, F. Uniqueness and nonuniqueness of bounded solutions to singular nonlinear parabolic equations. Nonlinear Anal. 70 (2009), 3020-3029. Z Zbl 1170.35463 MR 2509388 